\title{
Littered Cigarette Butts: A Grave Environmental Concern
}

Dipayan D*

BDS, Department of Public Health Dentistry, Tamil Nadu Government Dental College

and Hospital, India

*Corresponding author: Dipayan D, BDS, Department of Public Health Dentistry, Tamil Nadu Government Dental College and Hospital, Tamil Nadu, India, 600 003, Tel:

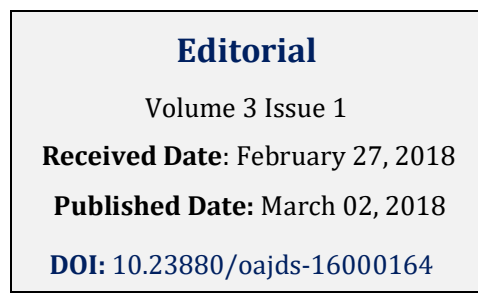

7358565184; E-mail: datta.dipayan2@gmail.com

\section{Editorial}

How often does a smoker think twice before littering the cigarette butts here and there? Should he care about that? What kind of harm would these little remnants create? Well the reality becomes quite different when studies show that around 4.5 trillion cigarette butts are littered worldwide every year by more than one billion smokers that generate approximately 2 billion pound waste over the earth surface.

Most of the cigarette filters are made of cellulose acetate, a plastic material that takes considerable amount of time (1.5 years on average) to degrade in the environment. Cellulose acetate fibres which are very thin in diameter are packed tightly together to create a filter; they can look like cotton. Cigarette filters are specifically designed to trap tar and vapours of other toxic chemicals and to accumulate particulate smoke components.

When a cigarette is burnt, it emits nearly 4000 chemicals through the smoke. Most of them get accumulated in the filter. A cigarette residue contains extremely hazardous chemicals like cadmium, lead and arsenic. Even after the butt is discarded after smoking, it can smoulder for at least 3 hours continuing to generate the same toxic smoke which creates air pollution to a great extent.

Areas where huge number of butts are littered look dirty and attract more littering of other waste products. When it rains, these butts are carried via the stream of water directly to the nearby water bodies, be it pond, lake, river or even sea. As the butts take long time to degrade in water, the harmful chemicals trapped in the filter start leaching out and intoxicating the water. The polluted water becomes hazardous for consumption or other uses and it also creates harmful environment for the marine creatures. Cigarette butts have even been found in the stomach of the dead young birds, sea turtles and some other marine animals as they mistook the butts as food resulting in fatal consequences. Not only animals, even young children were reported to have ingested littered cigarette butts and developed serious health outcomes.

As mentioned earlier, a littered butt can be found smouldering for hours which has high potential to cause fire in its surroundings. Nearly $10 \%$ of fire deaths worldwide are attributed to smoking. Only in America, numerous forest fires occur yearly due the littered butts resulting in trolls of death and loss of properties which cost billions of dollars. Also the huge amount of smoke coming out of the wildfires again pollutes the air.

It is time to create massive awareness in general population. Only the environmentalists should not be engaged in its resolution. Authorities from various fields such as academics, health, law, societies should stretch their hands towards a combined approach to solve the issue. From the perspective of a Public Health Dentist, along with the tobacco cessation counselling, he can include the general awareness of quitting cigarette smoking and thereby stoppage of littering cigarette butts in the oral health education and oral health promotion programmes. Once the common people are made well educated about the harmful effects of tobacco on health as well as the tobacco-related environmental hazards, and then only the problem can be uprooted from the environment. 